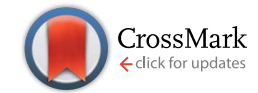

Cite this: RSC Adv., 2017, 7, 2745
Received 11th October 2016 Accepted 2nd December 2016

DOI: 10.1039/c6ra25052a

www.rsc.org/advances

\section{Carrier type change induced by fluorine doping in spin-chain compound $\mathrm{Ca}_{3} \mathrm{CO}_{2} \mathrm{O}_{6}$}

\author{
Jiyue Song, ${ }^{\text {ab }}$ Bangchuan Zhao, ${ }^{\text {a }}$ Yanan Huang, ${ }^{a}$ Yanfeng Qin, ${ }^{\text {ab }}$ Jiafeng Zhou, ${ }^{\text {ab }}$ \\ Wenhai Song ${ }^{a}$ and Yuping Sun ${ }^{\text {acd }}$
}

\begin{abstract}
The structural, magnetic, electrical, and thermoelectric properties of fluorine (F)-doped $\mathrm{Ca}_{3} \mathrm{CO}_{2} \mathrm{O}_{6}$ were investigated systematically. Based on the use of X-ray diffraction, X-ray photoelectron spectroscopy spectra, and magnetic data, it was concluded that part of the $\mathrm{Co}^{3+}$ ions at octahedral sites would transfer into $\mathrm{CO}^{2+}$ because of the substitution of $\mathrm{F}^{-}$for $\mathrm{O}^{2-}$. The induced $\mathrm{Co}^{2+}$ ions are antiferromagnetically coupled with the nearest neighboring $\mathrm{Co}^{3+}$. The room-temperature resistivity decreases monotonously with the increase of fluorine (F)-doping content, $x$, and it is suggested that this is related to the increased carrier mobility. The resistivity curves of the samples with $x \geq 0.4$ show an anomaly at $T^{*} \sim 320 \mathrm{~K}$, which is considered to be related to the change of activation energy $\left(E_{\mathrm{a}}\right)$. The room temperature thermopower $S_{300} \mathrm{k}$ changed from a positive value of $686.6 \mu \mathrm{V} \mathrm{K} \mathrm{K}^{-1}$ to a negative value of $-333.1 \mu \mathrm{V} \mathrm{K}^{-1}$ as $x$ increases from 0 to 0.4 and considering this with the Hall coefficient result, it is concluded that suitable $\mathrm{F}$-doping in $\mathrm{Ca}_{3} \mathrm{CO}_{2} \mathrm{O}_{6}$ can change the type of the majority of carriers from $\mathrm{p}$ to $n$ with a decreased resistivity. The thermal conductivity, $\kappa$ of the $n$-type samples is much larger than that of the $\mathrm{p}$-type, and the $\kappa$ of both the $\mathrm{p}$-type and $\mathrm{n}$-type samples decreases monotonously with increasing $x$. The anomaly of $\kappa$ near $x=0.4$ is suggested to originate from the decreased grain boundary scattering and the sudden decrease of $E_{\mathrm{a}}$ at the critical point of the carrier type change.
\end{abstract}

\section{Introduction}

Thermoelectric (TE) materials can be used to interconvert thermal energy and electric energy directly through the Seebeck effect or the Peltier effect in a single device. ${ }^{1}$ In general, the ideal TE materials need high thermopower $(S)$, low resistivity $(\rho)$ and thermal conductivity $(\kappa)$ to reach a high figure of merit $(\mathrm{ZT}=$ $\left.T S^{2} / \rho \kappa\right)$. Since the discovery of good TE performance in the layered structure cobaltites: $\mathrm{Na}_{x} \mathrm{Co}_{2} \mathrm{O}_{4},{ }^{2} \mathrm{Bi}_{2} \mathrm{Sr}_{2} \mathrm{Co}_{2} \mathrm{O}_{y},{ }^{3}$ and $\mathrm{Ca}_{3} \mathrm{Co}_{4} \mathrm{O}_{9}{ }^{4}$ the oxide TE materials have attracted much attention in recent years. Compared with these layered cobalt oxides ( $S \sim 100-125 \mu \mathrm{V} \mathrm{K}^{-1}$ at $300 \mathrm{~K}$ ), the quasi-one-dimensional cobalt oxide, $\mathrm{Ca}_{3} \mathrm{Co}_{2} \mathrm{O}_{6}$, has an extremely large thermopower ( $S \sim 680 \mu \mathrm{V} \mathrm{K}^{-1}$ at $300 \mathrm{~K}$ ) and excellent thermal stability ${ }^{5}$ (up to $1300 \mathrm{~K})$ in air.

Crystallization in a $\mathrm{K}_{4} \mathrm{CdCl}_{6}$ type structure with a space group $R \overline{3} c, \mathrm{Ca}_{3} \mathrm{Co}_{2} \mathrm{O}_{6}$ consists of infinite chains with alternating face

${ }^{a}$ Key Laboratory of Materials Physics, Institute of Solid State Physics, Chinese Academy of Sciences, Hefei 230031, People's Republic of China. E-mail: bchzhao@issp.ac.cn; Tel: +86-551-6559-2757

${ }^{b}$ University of Science and Technology of China, Hefei 230026, People's Republic of China

${ }^{c}$ High Magnetic Field Laboratory, Chinese Academy of Sciences, Hefei 230031, People's Republic of China

${ }^{d}$ Collaborative Innovation Center of Advanced Microstructures, Nanjing University, Nanjing 210093, People's Republic of China sharing $\mathrm{CoO}_{6}$ trigonal prisms and $\mathrm{CoO}_{6}$ octahedra along the $c$ axis. ${ }^{6}$ The chains form a triangular lattice in the $a b$ plane, separated by $\mathrm{Ca}^{2+}$ ions. The cobalt ions located at both the trigonal prism $\left(\mathrm{Co}_{\mathrm{I}} ; S=2\right)$ and octahedral sites $\left(\mathrm{Co}_{\mathrm{II}} ; S=0\right)$ are trivalent, ${ }^{7}$ while they are in different spin states because of the different crystalline electric field. Except for the interesting magnetic phenomena, such as magnetization steps, ${ }^{8}$ magnetoresistance $^{\mathbf{9}}$ and magnetodielectric coupling behavior ${ }^{\mathbf{1 0 , 1 1}}$ observed in $\mathrm{Ca}_{3} \mathrm{Co}_{2} \mathrm{O}_{6}$ and its derivative compounds, its $\mathrm{TE}$ properties have also attracted much attention in recent years. Since the high TE performance was reported by Mikami ${ }^{5}$ and Takahashi ${ }^{12}$ for a single crystal $\mathrm{Ca}_{3} \mathrm{Co}_{2} \mathrm{O}_{6}$, much research has been undertaken to improve its TE performance using element substitution or by optimizing the preparation method. The substitution of Fe for Co can lead to the enhancement of $S$ because of the change of spin and valence states of the Co ions. ${ }^{13}$ The $S$ at $800 \mathrm{~K}$ can be improved more than $400 \%$ using $\mathrm{Bi} / \mathrm{Cu}^{14}$ or $\mathrm{Na} / \mathrm{Mn}^{15}$ co-doping. In general, although $\mathrm{Ca}_{3} \mathrm{Co}_{2} \mathrm{O}_{6}$ is considered to be a p-type TE material, its carrier type can be changed to n-type using $5 \%$ Ti doping at the Co site. ${ }^{16}$ However, there is an undesirable effect of an increase of resistivity, which is not beneficial for a TE material. From the perspective of future applications, searching for an n-type $\mathrm{Ca}_{3} \mathrm{Co}_{2} \mathrm{O}_{6}$ with a low resistivity is imperative. Furthermore, the investigations on the effect of substitution in the $\mathrm{Ca}_{3} \mathrm{Co}_{2} \mathrm{O}_{6}$ system are mainly focused on the $\mathrm{Co}^{10,17}$ or $\mathrm{Ca}$ sites, ${ }^{18,19}$ and studies on the 
substitution effect at the $\mathrm{O}$ site have not been reported so far. To fully understand the element substitution effect on the physical properties of $\mathrm{Ca}_{3} \mathrm{Co}_{2} \mathrm{O}_{6}$, in this work the structural, magnetic and $\mathrm{TE}$ properties of $\mathrm{F}$-doped $\mathrm{Ca}_{3} \mathrm{Co}_{2} \mathrm{O}_{6}$ were systematically studied over a large $\mathrm{F}$ composition range $(0 \leq x \leq 0.8)$. The results show that an expected $\mathrm{Ca}_{3} \mathrm{Co}_{2} \mathrm{O}_{6}$-based n-type TE material was obtained using suitable F-doping. The carrier type changed from $\mathrm{p}$-type to $\mathrm{n}$-type as the F-doping level reached $x=$ 0.4 and the resistivity decreased monotonously with increasing F content. Although the ZT value of the series materials does not meet the standard of application $(\mathrm{ZT}>1)$, this work may give guidance for the study of other TE materials.

\section{Experimental details}

Polycrystalline samples of $\mathrm{Ca}_{3} \mathrm{Co}_{2} \mathrm{O}_{6-x} \mathrm{~F}_{x}(x=0-0.8)$ were prepared using a conventional solid-state reaction method. ${ }^{20}$ High purity powders of calcium carbonate $\left(\mathrm{CaCO}_{3}\right)$ cobalt(II,III) oxide $\left(\mathrm{Co}_{3} \mathrm{O}_{4}\right)$ and calcium fluoride $\left(\mathrm{CaF}_{2}\right)$ in a stoichiometric ratio were mixed thoroughly and preheated at $1223 \mathrm{~K}$ for $24 \mathrm{~h}$ twice with an intermediate grinding step. Then the mixtures obtained were reground, pressed into circular pellets, and sintered at $1223 \mathrm{~K}$ for another $48 \mathrm{~h}$ in air. The structure and phase purity of the samples were checked using powder X-ray diffraction (XRD) using a Philips X'pert PRO X-ray diffractometer with $\mathrm{Cu} \mathrm{K}_{\alpha}$ radiation $\left(20^{\circ} \leq 2 \theta \leq 80^{\circ}\right)$ at room temperature. The X-ray photoelectron spectra (XPS) were obtained using a Thermo Scientific ESCALAB 250 spectrometer using Al K $\alpha$ Xrays at $1486.6 \mathrm{eV}$ as the excitation source. The microstructures were investigated using a scanning electron microscope (SEM). The chemical composition was determined on an energy dispersive spectroscope (EDS) attached to the SEM equipment. The magnetic measurements were carried out with a superconducting quantum interference device (SQUID) magnetic property measurement system (MPMS) system. The Hall coefficient $\left(R_{\mathrm{H}}\right)$ at room temperature and the temperature dependence of resistivity, thermopower, and thermal conductivity were all measured using a Quantum Design physical property measurement system (PPMS). The $R_{\mathrm{H}}$ data were obtained under a magnetic field range from $-4 \mathrm{~T}$ to $4 \mathrm{~T}$. The carrier concentration $\left(n^{*}\right)$ and carrier mobility $(\mu)$ were calculated according to $n^{*}=1 /\left(e R_{\mathrm{H}}\right)$ and $\sigma_{x x}=n^{*} e \mu$, respectively.

\section{Results and discussion}

\section{Structure}

The room temperature powder XRD patterns of $\mathrm{Ca}_{3} \mathrm{Co}_{2} \mathrm{O}_{6-x} \mathrm{~F}_{x}(x$ $=0-0.8$ ) samples are shown in Fig. 1(a). The results show that Fdoping did not change the lattice structure of this system. All samples were found to have the rhombohedral structure with space group $R \overline{3} c$. To clearly show the effect of F-doping on the lattice, the enlarged (300) peaks are shown in Fig. 1(b). As the Fdoping content increases, the (300) peak moves to a higher angle position first and then moves in the opposite direction. The XRD patterns were refined using the standard Rietveld technique and the lattice parameters obtained were plotted as a function of the doping level $(x)$ in the inset of Fig. 1(a). It was

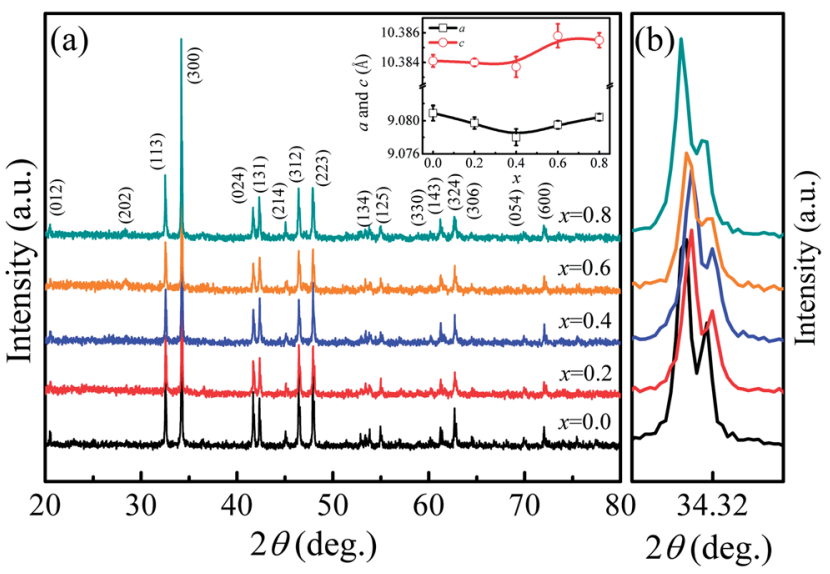

Fig. 1 (a) XRD patterns of $\mathrm{Ca}_{3} \mathrm{Co}_{2} \mathrm{O}_{6-x} \mathrm{~F}_{x}(x=0,0.2,0.4,0.6,0.8)$. The inset presents the lattice parameters as functions of the doping content $(x)$. (b) The enlarged (300) peaks for all samples.

found that both the parameters $a$ and $c$ decrease first and then increase with the increase of $x$, which is consistent with the shift of the (300) peaks as shown in Fig. 1(b).

In general, $\mathrm{F}^{-}$doping at the $\mathrm{O}^{2-}$ site should give rise to the decrease of lattice parameters because of the slightly smaller ionic radius of $\mathrm{F}^{-}(1.33 \AA)$ compared to that of $\mathrm{O}^{2-}(1.4 \AA)$, but this is inconsistent with the results observed in this work. In fact, as $\mathrm{O}^{2-}$ ions are substituted by $\mathrm{F}^{-}$, some of the $\mathrm{Co}^{3+}$ ions should change into $\mathrm{Co}^{2+}$ to keep the charge neutrality. The radius of $\mathrm{Co}^{2+}\left[0.65 \AA\right.$ for low spin (LS) $\mathrm{Co}^{2+}$ and $0.745 \AA$ for high spin (HS) $\left.\mathrm{Co}^{2+}\right]$ is larger than that of $\mathrm{Co}^{3+}\left(0.545 \AA\right.$ for $\mathrm{HS} \mathrm{Co}^{3+}$ and $0.61 \AA$ for $\mathrm{LS} \mathrm{Co}^{3+}$ ), and the ion transfer induced by $\mathrm{F}$ doping will lead to the expansion of lattice. The variation of the lattice parameters found in the present work was affected by the two competing factors discussed previously. The role of the two factors was different as the F-doping level changed. As the Fdoping is slight, the substituted $\mathrm{F}^{-}$ions with smaller ionic radius lead to the decreased lattice parameters shown in the inset of Fig. 1(a). In the heavily F-doped samples, the increased lattice parameter should be dominated by the ionic radius difference between $\mathrm{Co}^{3+}$ and the induced $\mathrm{Co}^{2+}$ ions.

In order to show the valence transition of Co ions clearly, XPS measurements were performed on two typical samples with $x=$ 0 and 0.8, and the results are shown in Fig. 2(a) and (b), respectively. As Fig. 2(a) shows, two distinct peaks located at $\sim 780 \mathrm{eV}$ and $\sim 796 \mathrm{eV}$ correspond to the Co 2 p core-level spectrum of $\mathrm{Ca}_{3} \mathrm{Co}_{2} \mathrm{O}_{6},{ }^{21}$ and the weak peaks situated at $\sim 789.1 \mathrm{eV}$ and $\sim 804.3 \mathrm{eV}$ are the satellite peaks of Co ions. ${ }^{22}$ The peak located at $\sim 780 \mathrm{eV}$ can be deconvoluted into two peaks. The two peaks corresponding to the higher binding energy (BE) and the lower $\mathrm{BE}$ are attributed to $\mathrm{Co}^{2+}$ and $\mathrm{Co}^{3+}$ ions, respectively. The $\mathrm{BE}$ of the $\mathrm{Co}^{3+} 2 \mathrm{p}_{3 / 2}$ peak located at $780.4 \mathrm{eV}$ is close to that of $\mathrm{Co}^{3+} 2 \mathrm{p}_{3 / 2}$ in $\mathrm{ZnCo}_{2} \mathrm{O}_{4}(780.6 \mathrm{eV}){ }^{23}$ and the $\mathrm{BE}$ of the $\mathrm{Co}^{2+} 2 \mathrm{p}_{3 / 2}$ peak located at $782.2 \mathrm{eV}$ is close to that of $\mathrm{Co}^{2+} 2 \mathrm{p}_{3 / 2}$ in $\mathrm{Co}(\mathrm{OH})_{2}$ $(782 \mathrm{eV}) .{ }^{24}$ Similarly, the deconvoluted peaks located at $795.5 \mathrm{eV}$ and $798 \mathrm{eV}$ correspond to $\mathrm{Co}^{3+} 2 \mathrm{p}_{1 / 2}$ and $\mathrm{Co}^{2+} 2 \mathrm{p}_{1 / 2} \cdot{ }^{22}$ As can be seen from the figure, a small amount of $\mathrm{Co}^{2+}$ ions existed in the un-doped sample of $\mathrm{Ca}_{3} \mathrm{Co}_{2} \mathrm{O}_{6}$ and the ratio of $\mathrm{Co}^{2+}$ is estimated 


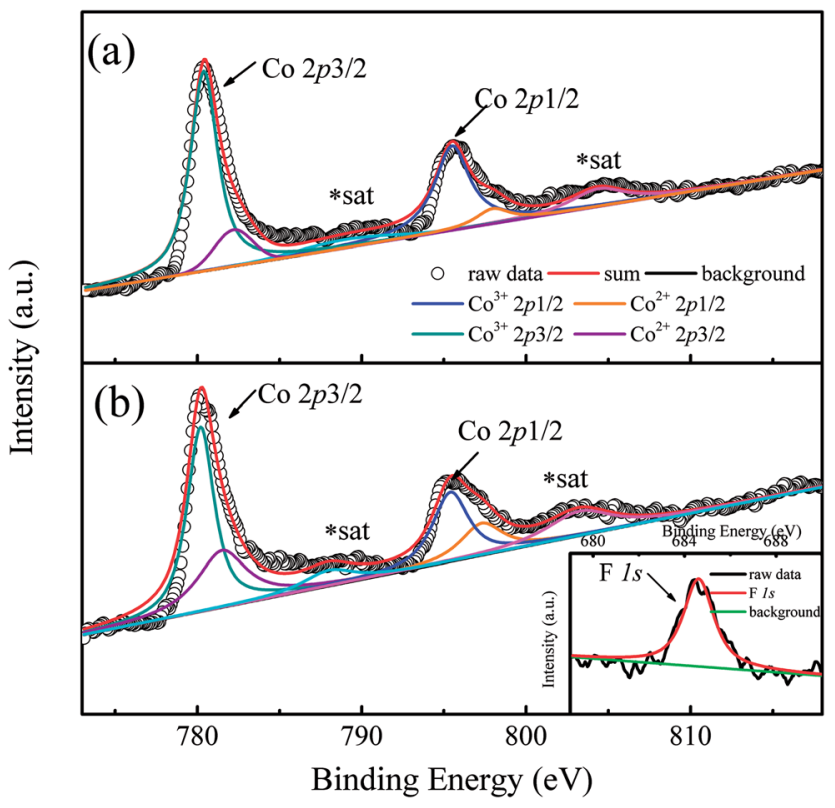

Fig. 2 (a) $\mathrm{Co} 2 \mathrm{p}$ photoelectron spectrum of $\mathrm{Ca}_{3} \mathrm{Co}_{2} \mathrm{O}_{6}$. (b) $\mathrm{Co} 2 \mathrm{p}$ photoelectron spectrum of $x=0.8$ sample. Inset shows the $\mathrm{F} 1 \mathrm{~s}$ peak.

at $12 \%$. In general, the $\mathrm{Co}$ ions of $\mathrm{Ca}_{3} \mathrm{Co}_{2} \mathrm{O}_{6}$ at both trigonal prismatic and octahedral sites are in the trivalent state.$^{25}$ The presence of a small amount of $\mathrm{Co}^{2+}$ in the un-doped sample should be related to the oxygen non-stoichiometry induced during the sample preparation process. Compared with Fig. 2(a) and (b), the area of the deconvoluted $\mathrm{Co}^{2+} 2 \mathrm{p}_{3 / 2}$ and $\mathrm{Co}^{2+} 2 \mathrm{p}_{1 / 2}$ peaks increases obviously as the $\mathrm{F}^{-}$ions go into the system. The calculated content of $\mathrm{Co}^{2+}$ ions is about $35 \%$ in the sample with $x=0.8$, implying that part of the $\mathrm{Co}^{3+}$ ions transfer into $\mathrm{Co}^{2+}$ via the F-doping. The increased content of $\mathrm{Co}^{2+}$ gives rise to the increase of lattice parameters because of its much larger ionic radius than that of $\mathrm{Co}^{3+}$ as previously mentioned.

\section{Magnetic properties}

The temperature dependence of magnetization $M(T)$ for $\mathrm{Ca}_{3}$ $\mathrm{Co}_{2} \mathrm{O}_{6-x} \mathrm{~F}_{x}$ was measured in the temperature range of $3-150 \mathrm{~K}$ and the data below $50 \mathrm{~K}$ are shown in Fig. 3(a)-(e). The temperature dependence of inverse dc susceptibility fitted using the Curie-Weiss law are shown in the insets of Fig. 3(a)-(e). The $M(T)$ data were collected in zero-field-cooling (ZFC), fieldcooling (FC), and field-cooling-warming (FCW) modes at an applied field of 100 Oe. Only the ZFC and FCW curves are shown in this figure because the FCC and FCW curves almost overlap. From Fig. 3(a)-(e), it can be seen that all samples undergo a long-range spin density wave transition ${ }^{26-28}$ at $T_{\mathrm{c} 1}$ and a spinglass like transition ${ }^{29}$ at $T_{\mathrm{c} 2}$ with the decrease of temperature. Fdoping does not obviously change the two magnetic transitions, but the value of the low temperature magnetization decreases with the increase of F-doping content.

The effective magnetic moment ( $\left.\mu_{\text {eff }}\right)$ obtained as a function of F-doping level $x$ was plotted and is shown in Fig. 3(f). As Fig. 3(f) shows, $\mu_{\text {eff }}$ increases monotonously with the increasing $x$. As discussed previously, the substitution of $\mathrm{F}^{-}$for $\mathrm{O}^{2-}$ should lead to part of the $\mathrm{Co}^{3+}$ ions to transfer into $\mathrm{Co}^{2+}$. In general, ${ }^{7}$ the Co ions in the $\mathrm{Ca}_{3} \mathrm{Co}_{2} \mathrm{O}_{6}$ system prefer to be in the HS state at trigonal prism sites $\left(\mathrm{Co}_{\mathrm{I}}\right)$, whereas the Co ions located at the octahedral sites $\left(\mathrm{Co}_{\text {II }}\right)$ are in the LS state because of the different crystalline field. If F-doping leads to $\mathrm{Co}^{3+}$ ions at $\mathrm{Co}_{\text {I }}$ sites (HS; $S$ $=2$ ) transferring into $\mathrm{Co}^{2+}$ ions (HS; $S=3 / 2$ ), the value of $\mu_{\text {eff }}$ should decrease with the increase of $x$, which is contrary to the experimental data obtained. So it was concluded that the ionic transfer induced by F-doping occurred at $\mathrm{Co}_{\text {II }}$ sites, leading to the increase of $\mu_{\text {eff. }}$ The theoretically expected (spin only) values of the $\mu_{\text {eff }}$ in $\mathrm{Ca}_{3} \mathrm{Co}_{2} \mathrm{O}_{6}$ system can be expressed as:

$$
\mu_{\mathrm{eff}}=g \sqrt{S_{\mathrm{Co}_{\mathrm{I}}}\left(S_{\mathrm{Co}_{\mathrm{I}}}+1\right)} \mu_{\mathrm{B}}
$$

where $g, S_{\mathrm{Co}_{\mathrm{I}}}, \mu_{\mathrm{B}}$ are the Landé factor, spin quantum number of Co ions at $\mathrm{Co}_{\mathrm{I}}$ sites, and Bohr magneton, respectively. If it is considered that the ionic transfer for Co ions only occurred at $\mathrm{Co}_{\text {II }}$ sites, the equation can be rewritten as: ${ }^{30}$

$$
\mu_{\mathrm{eff}}=\left[g \sqrt{S_{\mathrm{Co}_{\mathrm{I}}}\left(S_{\mathrm{Co}_{\mathrm{I}}}+1\right)}+\gamma g \sqrt{S_{\mathrm{Co}_{\mathrm{II}}}\left(S_{\mathrm{Co}_{\mathrm{II}}}+1\right)}\right] \mu_{\mathrm{B}},
$$

where $\gamma$ is the ratio of the transferred $\mathrm{Co}^{3+}$ ions at $\mathrm{Co}_{\text {II }}$ sites $(S=$ 0 to $S=1 / 2$ ). The calculated $\mu_{\text {eff }}$ based on eqn (2) is plotted in Fig. 3(f). For the un-doped sample, the $\mu_{\text {eff }}$ obtained using Curie-Weiss fitting is $5.5 \mu_{\mathrm{B}} \mathrm{fu}^{-1}$, which is consistent with the previous reported value $\left(5.7 \pm 0.2 \mu_{\mathrm{B}} \mathrm{fu}^{-1}\right) .{ }^{31}$ The experimental value of $\mu_{\text {eff }}$ found in the present work is larger than the $\mu_{\text {eff }}$ calculated using eqn (2), which may be related to the orbital moment originating from the spin-orbital coupling and an unusual coordination of the $\mathrm{Co}$ ions at $\mathrm{Co}_{\mathrm{I}}$ sites as reported by Wu et al. $^{32}$ The difference between the experimental and theoretical $\mu_{\text {eff }}$ value decreases considerably with the increase of $x$, indicating that the orbital moment can be suppressed effectively by $\mathrm{F}^{-}$doping. The Weiss temperature $\theta$ obtained from the Curie-Weiss fitting of all samples were positive, indicating that the dominated magnetic interaction is ferromagnetic. The value of $\theta$ decreases monotonically with increasing $x$, indicating that the induced $\mathrm{Co}^{2+}$ ions ( $\mathrm{Co}_{\text {II }}$ sites) may be antiferromagnetically coupled with the $\mathrm{Co}^{3+}$ ions at the $\mathrm{Co}_{\mathrm{I}}$ site.

To thoroughly understand the F-doping effect on the magnetic properties, the magnetic field dependent magnetization $(M-H)$ was measured at $3 \mathrm{~K}$ and the results are shown in Fig. 4. As shown in Fig. 4(a)-(e), all the samples show a large hysteresis in the $M-H$ curve, and the magnetization does not become saturated as the magnetic field reaches $4.5 \mathrm{~T}$. There are three magnetization steps which occurred at 1.2, 2.4, and 3.6 $\mathrm{T}$ in the initial magnetization curve of $\mathrm{Ca}_{3} \mathrm{Co}_{2} \mathrm{O}_{6}$, which is related to the quantum tunneling of the magnetization behavior. ${ }^{8}$ Furthermore, the magnetization step at $3.6 \mathrm{~T}$ can be considered further as the formation of a ferrimagnetic state consisting of two-thirds of spin-up ferromagnetic chains and one-third of spin-down chains. To show the evolution of these magnetization steps with F-doping clearly, three dashed lines near these steps are plotted in the figure. Different to the doping effect on $\mathrm{Ca}^{18}$ or Co-sites, ${ }^{30}$ F-doping at the O-site does not obviously change the magnetization steps, and the position of the magnetization steps were almost the same for all the samples 

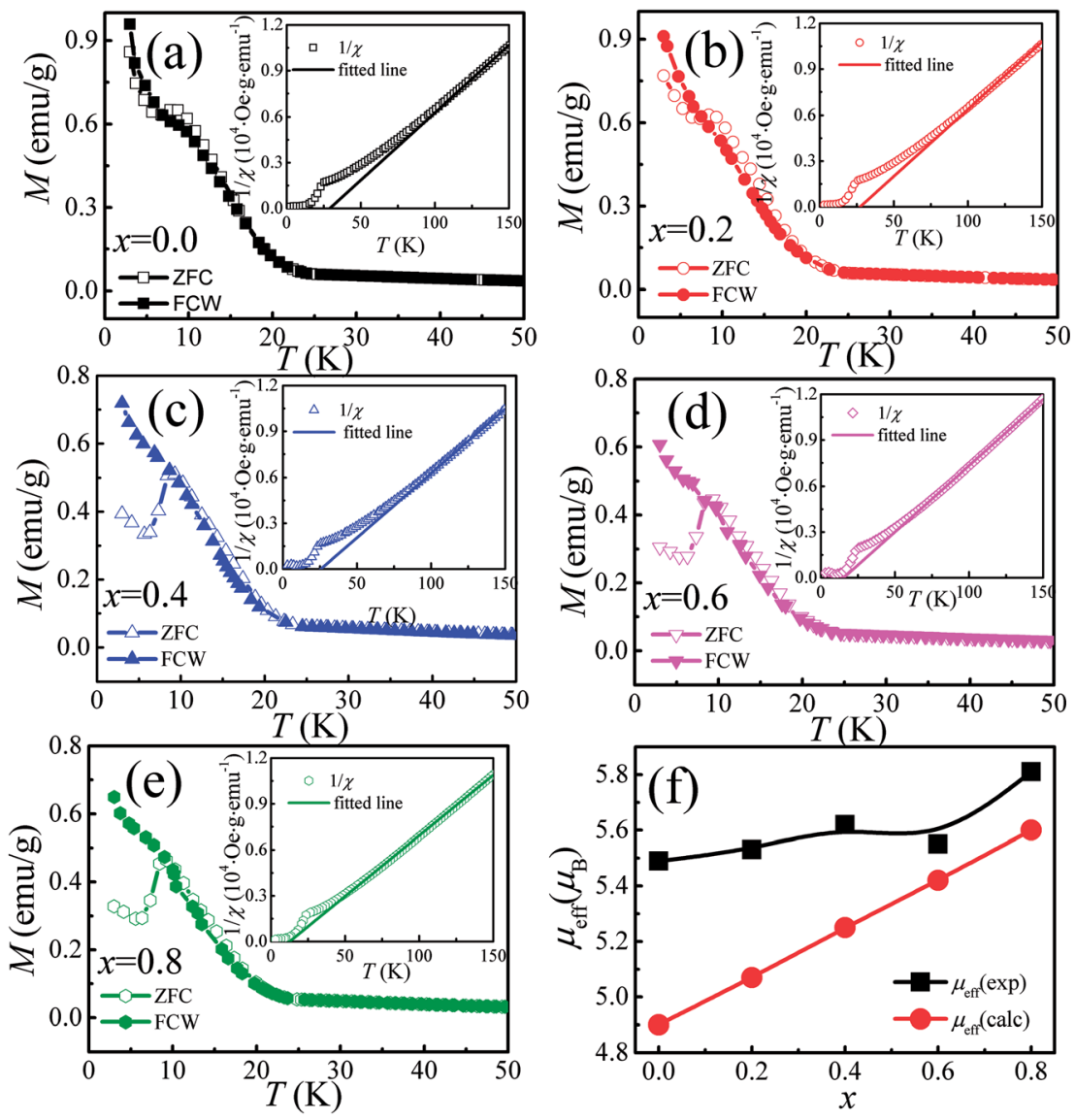

Fig. 3 (a)-(e) Temperature dependence of dc magnetization under 100 Oe for $\mathrm{Ca}_{3} \mathrm{CO}_{2} \mathrm{O}_{6-x} \mathrm{~F}$. The open and solid symbols correspond to ZFC and FC curves, respectively. The inset shows the temperature dependence of the inverse dc susceptibility, and the solid lines are the fitting curves obtained using the Curie-Weiss law. (f) The theoretical and experimental $\mu_{\text {eff }}$ versus F-doping content $x$.

studied. Although F-doping has little influence on the position of these magnetization steps, the magnetization value of each step decreases with the increase of F-doping content, as shown in Fig. 4(f). As is known, the magnetization existing in this spinchain compound is a spin and lattice sensitive phenomenon. In the present work, the F-doping does not induce additional spins and the lattice distortion caused by the substitution of $\mathrm{F}$ for $\mathrm{O}$ is small because of the small ionic radius difference between $\mathrm{F}^{-}$ $(1.33 \AA)$ and $\mathrm{O}^{2-}$ ions $(1.4 \AA)$. So the F-doping has a weak influence on these magnetization steps.

\section{Electrical transport properties}

The temperature dependence of the resistivity for $\mathrm{Ca}_{3} \mathrm{Co}_{2} \mathrm{O}_{6-x} \mathrm{~F}_{x}$ in the temperature range of $170-380 \mathrm{~K}$ is shown in Fig. 5(a). All the samples show a similar transport behavior in the temperature range measured. To show the resistivity variation with Fdoping clearly, the room temperature resistivity $\rho_{300} \mathrm{~K}$ values are listed in Table 1 . The room temperature resistivity decreases monotonously with increasing $x$. In general, the substitution of $\mathrm{F}^{-}$for $\mathrm{O}^{2-}$ is considered to be n-type doping, and the carrier concentration should decrease with increasing $x$ for a typical ptype semiconductor, $\mathrm{Ca}_{3} \mathrm{Co}_{2} \mathrm{O}_{6}$, which is contrary to the experimental results discussed here. To study the origin of the variation of $\rho_{300 \mathrm{~K}}$, the Hall measurement was performed for all samples at $300 \mathrm{~K}$. The room temperature Hall coefficient $\left(R_{\mathrm{H}}\right)$ as a function of $x$ is shown in the inset of Fig. 5(a), and the calculated values of carrier concentration $\left(n^{*}\right)$ and carrier mobility $(\mu)$ are listed in Table 1 . It was found that $R_{\mathrm{H}}$ is changed from positive to negative when $x$ increased to 0.4 , indicating that the carrier type has changed from a p-type to a n-type. The calculated carrier concentration $n^{*}$ first decreases with increasing $x$ up to 0.2 , and then has an increasing trend as $x$ increases further. Unlike the carrier concentration, the mobility of the carrier has an increasing trend with increasing $x$ in the whole range of F-doping levels.

In order to clarify the transport mechanism of F-doped $\mathrm{Ca}_{3} \mathrm{Co}_{2} \mathrm{O}_{6}$, fitting of the resistivity data was attempted using the thermal activated (TAC) law: ${ }^{33} \rho=\rho_{\infty} \exp \left(E_{0} / k_{\mathrm{B}} T\right)$, the adiabatic small polaron hopping model: ${ }^{34} \rho=A T \exp \left(E_{\mathrm{\rho}} / k_{\mathrm{B}} T\right)$, and the Mott variable range hopping model: ${ }^{35} \rho=\rho_{0} \exp \left[\left(T_{0} / T\right)^{1 /(1+n)}\right]$, respectively. For the samples with $x \leq 0.2$ (p type), the electrical transport behaviors were described well by the TAC model over the whole of the measured temperature range. Whereas for the samples with $x=0.4,0.6$ and 0.8 (n type), the $\rho(T)$ curves were fitted well using the TAC models with different activation energies $\left(E_{\mathrm{a}}\right)$ in two different temperature ranges divided by $T^{*} \sim 320 \mathrm{~K}$. The fitting results are shown in Fig. 5(b)-(f) and the results for the parameter $E_{\mathrm{a}}$ are listed in Table 1. 

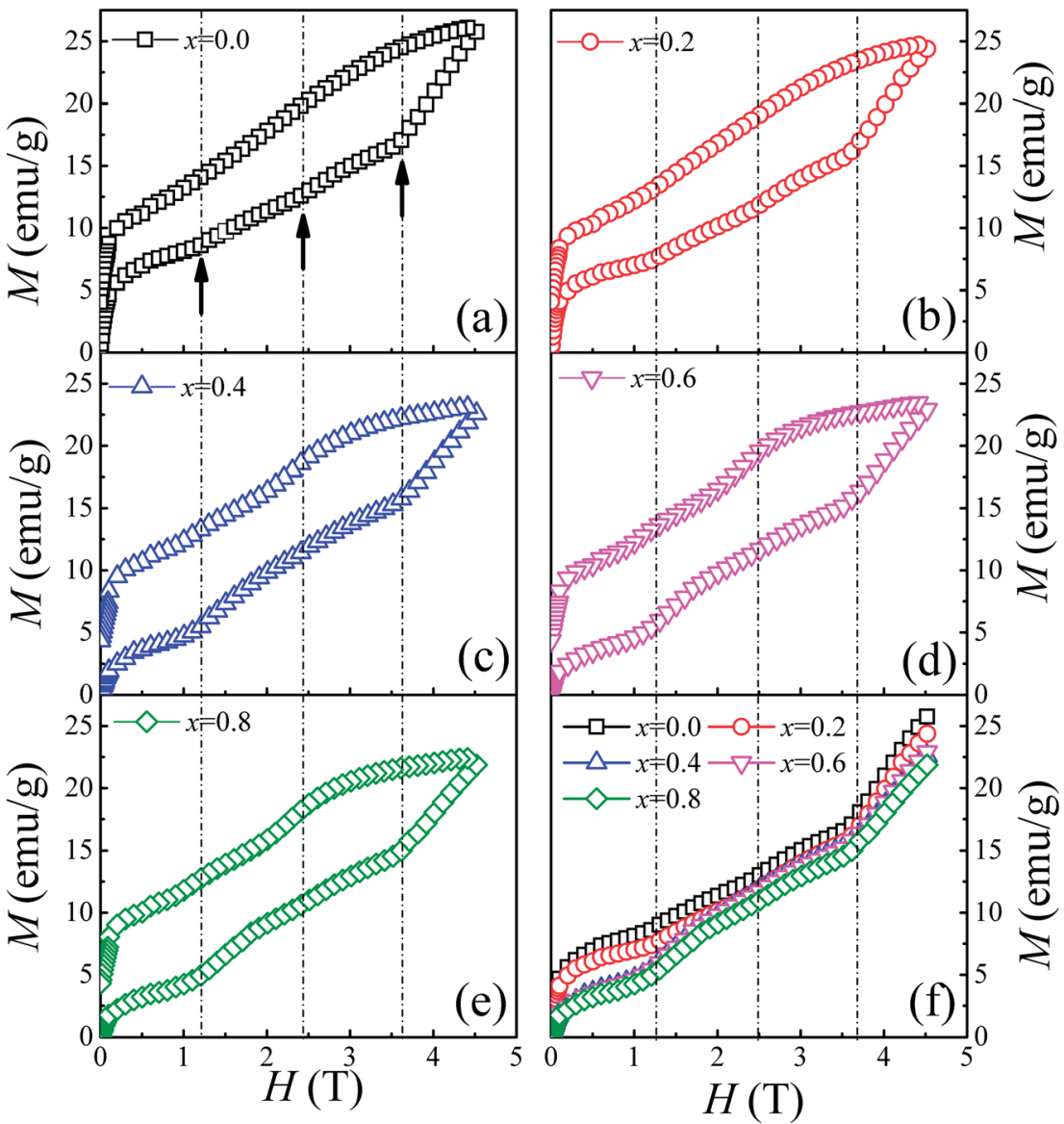

Fig. 4 (a)-(e) Field dependence of magnetization at $3 \mathrm{~K}$ for $\mathrm{Ca}_{3} \mathrm{CO}_{2} \mathrm{O}_{6-x} \mathrm{~F}_{x}(x=0,0.2,0.4,0.6,0.8)$. (f) The initial magnetization curves for $\mathrm{Ca}_{3} \mathrm{CO}_{2} \mathrm{O}_{6-x} \mathrm{~F}_{x}$.

Fig. 6(a)-(e) show the SEM images of the cross-section of the samples with $x=0,0.2,0.4,0.6$, and 0.8 . It can be seen that the surface morphologies for all the samples studied are similar, whereas the particle size increases with increasing $x$. The mean particle size is estimated from 10 randomly selected particles in the SEM images for each sample. It was found that the values of mean particle size $(d)$ were $2.26,2.46,2.65,3.30$, and $3.90 \mu \mathrm{m}$ for the samples with $x=0,0.2,0.4,0.6$, and 0.8 , respectively. In addition, the particles become more homogenous and denser with the increase of $x$. The much larger particle size and denser microstructure in the F-doped samples will promote carrier transport because of the decreased grain boundary scattering and thus the carrier mobility will be enhanced, which was also confirmed by the Hall measurements as mentioned previously. The enhanced carrier mobility may be a key factor for the decrease of resistivity in the F-doped samples. Fig. 6(f) shows the EDS results for a typical sample with $x=0.8$. This confirms the presence of $\mathrm{Ca}, \mathrm{Co}, \mathrm{O}, \mathrm{F}$ elements and the average atomic ratios are $\mathrm{Ca}: \mathrm{Co}: \mathrm{O}: \mathrm{F}=3: 1.97: 4.95: 0.72$ when setting the content of $\mathrm{Ca}$ as 3 . This is roughly consistent with the chemical formula of $\mathrm{Ca}_{3} \mathrm{Co}_{2} \mathrm{O}_{5.2} \mathrm{~F}_{0.8}$.

\section{Thermoelectric properties}

Fig. 7 shows the temperature dependence of thermopower $[S(T)]$ for $\mathrm{Ca}_{3} \mathrm{Co}_{2} \mathrm{O}_{6} \mathrm{~F}_{x}$ samples. The $x=0$ and $x=0.2$ samples have a positive value of $S$ in the whole of the temperature range studied and the sign of $S$ changed to negative as the F-doping content reached 0.4. The results show that the carrier type can be changed from p-type to n-type using a suitable amount of Fdoping. The absolute values of $S$ for all samples show a similar trend in variation: $S$ increases first and then decreases with increasing temperature. The room temperature thermopower $S_{300 \mathrm{~K}}$ for the un-doped sample is $686.6 \mu \mathrm{V} \mathrm{K}{ }^{-1}$, which is consistent with a previous report. ${ }^{36}$ With increasing $x, S_{300 \mathrm{~K}}$ increases from $686.6 \mu \mathrm{V} \mathrm{K}^{-1}(x=0)$ to $752.7 \mu \mathrm{V} \mathrm{K}^{-1}(x=0.2)$ and then changes from $-333.1 \mu \mathrm{V} \mathrm{K}^{-1}(x=0.4)$ to $-224.7 \mu \mathrm{V} \mathrm{K}^{-1}(x$ $=0.8$ ).

For a general semiconducting material, $S$ can be expressed using the Mott formula: ${ }^{37}$

$$
S=\frac{\pi^{2} k_{\mathrm{B}}^{2} T}{3 e}\left[\frac{\partial \ln \sigma(\varepsilon)}{\partial \varepsilon}\right]_{\varepsilon=\varepsilon_{\mathrm{F}}}
$$

By substituting $\sigma=n^{*} e \mu(\varepsilon)$ in eqn (3), one can obtain:

$$
S=\frac{C_{\mathrm{e}}}{n^{*}}+\frac{\pi^{2} k_{\mathrm{B}}^{2} T}{3 e}\left[\frac{\partial \ln \mu(\varepsilon)}{\partial \varepsilon}\right]_{\varepsilon=\varepsilon_{\mathrm{F}}},
$$

where $C_{\mathrm{e}}, n^{*}, \mu(\varepsilon)$, and $k_{\mathrm{B}}$ are specific heat, carrier concentration, carrier mobility, and Boltzmann constant, respectively. In 


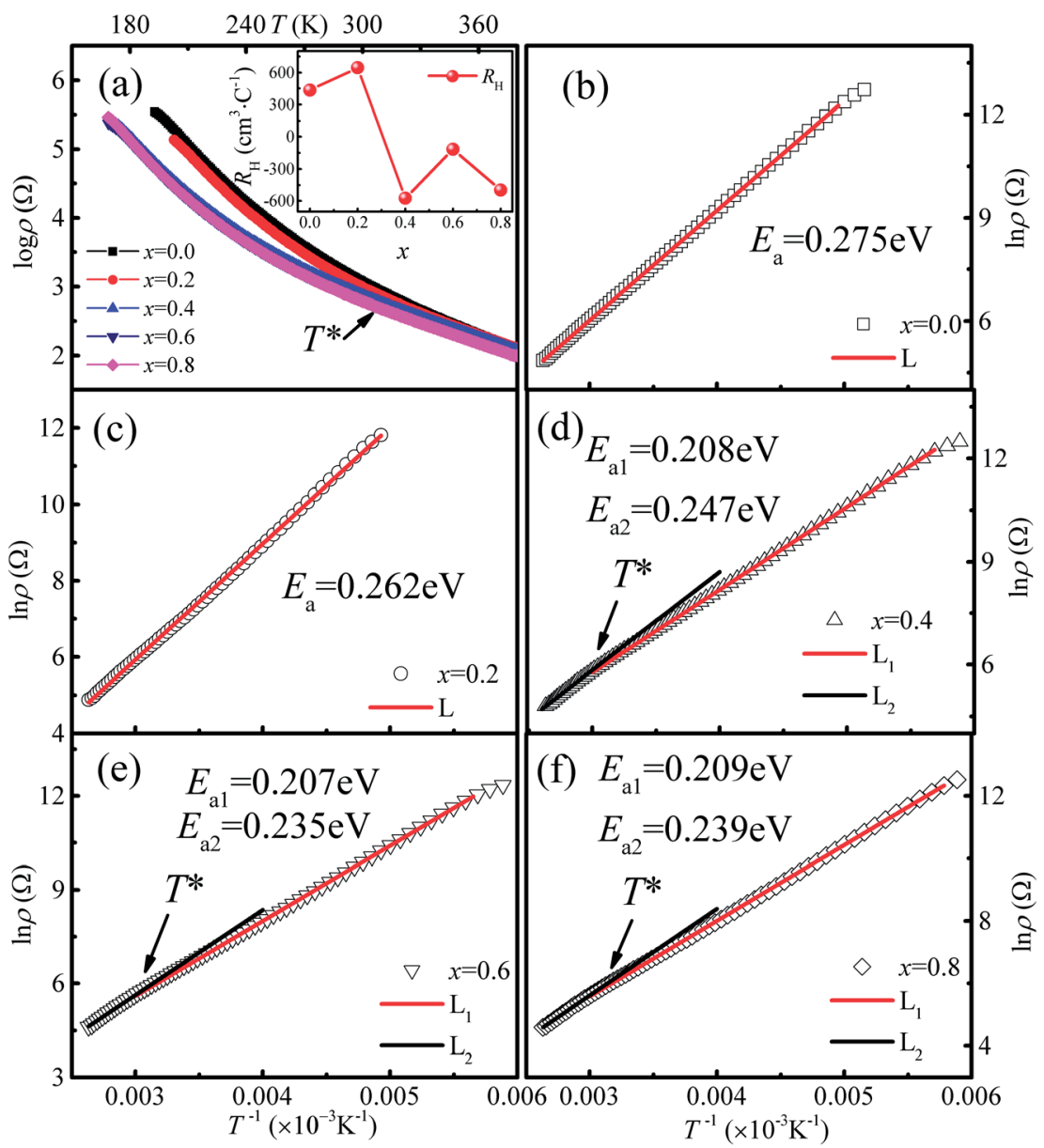

Fig. 5 (a) Temperature dependence of resistivity for $\mathrm{Ca}_{3} \mathrm{CO}_{2} \mathrm{O}_{6-x} \mathrm{~F}_{x}$. Inset shows the room temperature Hall coefficient $\left(R_{\mathrm{H}}\right)$ as a function of $x$. (bf) The TAC fitting results for the samples with $x=0-0.8$.

Table 1 The electrical and thermoelectric transport parameters of Fdoped $\mathrm{Ca}_{3} \mathrm{CO}_{2} \mathrm{O}_{6}$ samples

\begin{tabular}{lccccc}
\hline & $x=0.0$ & $x=0.2$ & $x=0.4$ & $x=0.6$ & $x=0.8$ \\
\hline$\rho_{300 \mathrm{~K}}(\Omega \mathrm{cm})$ & 1160.7 & 969.3 & 740.1 & 627.8 & 616.5 \\
$E_{\mathrm{a}}(\mathrm{eV})$ & 0.275 & 0.262 & 0.208 & 0.207 & 0.209 \\
$R_{\mathrm{H}}\left(10^{2} \mathrm{~cm}^{3} \mathrm{C}^{-1}\right)$ & 4.34 & 6.44 & -5.73 & -1.17 & -5.00 \\
$n^{*}\left(10^{16} \mathrm{~cm}^{-3}\right)$ & 1.44 & 0.94 & -1.09 & -5.33 & -1.25 \\
$\mu\left(10^{-1} \mathrm{~cm}^{2} \mathrm{~V}^{-1} \mathrm{~s}^{-1}\right)$ & 3.75 & 6.89 & 7.78 & 1.87 & 8.09 \\
$d(\mu \mathrm{m})$ & 2.26 & 2.46 & 2.65 & 3.30 & 3.90 \\
$S_{300 \mathrm{~K}}\left(\mu \mathrm{V}^{-1} \mathrm{~K}^{-1}\right)$ & 686.6 & 752.7 & -333.1 & -289.3 & -224.7 \\
$\Theta_{\mathrm{D}}(\mathrm{K})$ & 600.1 & 413.8 & 420.6 & 389.8 & 296.5
\end{tabular}

the present system, the thermopower is considered to be dominated by the first term of the above equation, which is similar to the simple Drude picture, $S \sim C_{\mathrm{e}} / n^{*}$. For the p-type samples with $x \leq 0.2$, the increase of $S_{300 \mathrm{~K}}$ is because of the decreased p-type carrier concentration caused by the substitution of $\mathrm{F}^{-}$for $\mathrm{O}^{2-}$. However, F-doping in the heavily doped samples will increase the carrier concentration because of its ntype transport mechanism, which is the origin of the decrease of the absolute value of $S_{300 \mathrm{~K}}$ for the samples with $x \geq 0.4$.
The temperature dependence of thermal conductivity $\kappa(T)$ is shown in Fig. 8. All the samples show a similar thermal transport behavior, but the value of $\kappa$ changed considerably as $\mathrm{F}$ was doped into the samples. $\kappa$ increases with the decrease of $T$ in the temperature range of $T>T_{\mathrm{p}}$, whereas $\kappa$ decreases sharply with decreasing $T$ when $T<T_{\mathrm{p}}$. As shown in the inset of Fig. 8(b), the values of $\kappa$ at $T_{\mathrm{p}}$ for both n-type and p-type samples decrease monotonously with increasing $x$, but there exists a sudden increase of thermal conductivity at the critical point where the carrier type changes from p- to n-type, which may be related to decreased grain boundary scattering and the sudden decrease of $E_{\mathrm{a}}$ when $x$ reaches 0.4 . Generally, thermal conductivity can be expressed by the sum of phonon thermal conductivity $\kappa_{\mathrm{ph}}$ and carrier thermal conductivity $\kappa_{\text {car }}$ as: $\kappa=\kappa_{\mathrm{ph}}+\kappa_{\mathrm{car}}$. The value of $\kappa_{\text {car }}$ can be calculated from the Wiedemann-Franz (WF) law: $\kappa_{\text {car }}$ $=L T / \rho$, where $L$ is the Lorentz number. The calculated $\kappa_{\text {car }}$ in the present system is less than $0.1 \%$. Therefore, the phonon contribution is the main source of total $\kappa$ in this system. The phonon conductivity can be described as $\kappa_{\mathrm{p}} \propto C_{\mathrm{v}} \lambda v_{0}$, where $C_{\mathrm{v}}$, $\lambda$, and $v_{0}$ are the specific heat, mean free path, and mean velocity of phonon, respectively. When $T>T_{\mathrm{p}}$, the phononphonon Umklapp scattering mechanism is dominant in determining the thermal transport behavior and $\lambda$ can be described 

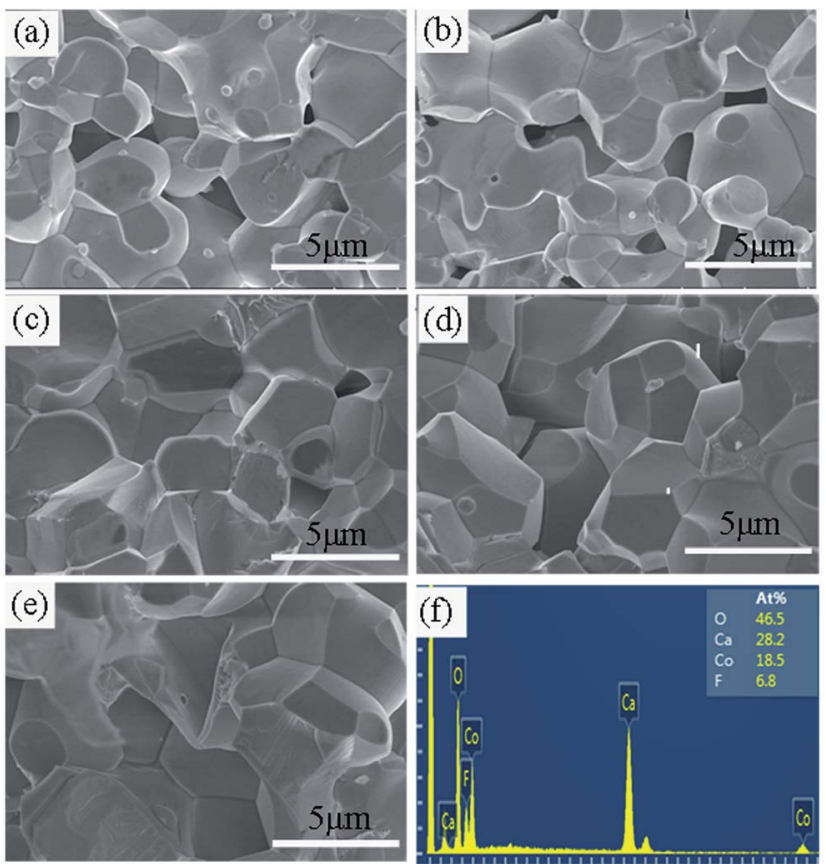

Fig. 6 (a-e) The SEM images for the samples with $x=0,0.2,0.4,0.6$, and 0.8 , respectively. (f) The EDS results for a typical sample with $x=$ 0.8 .

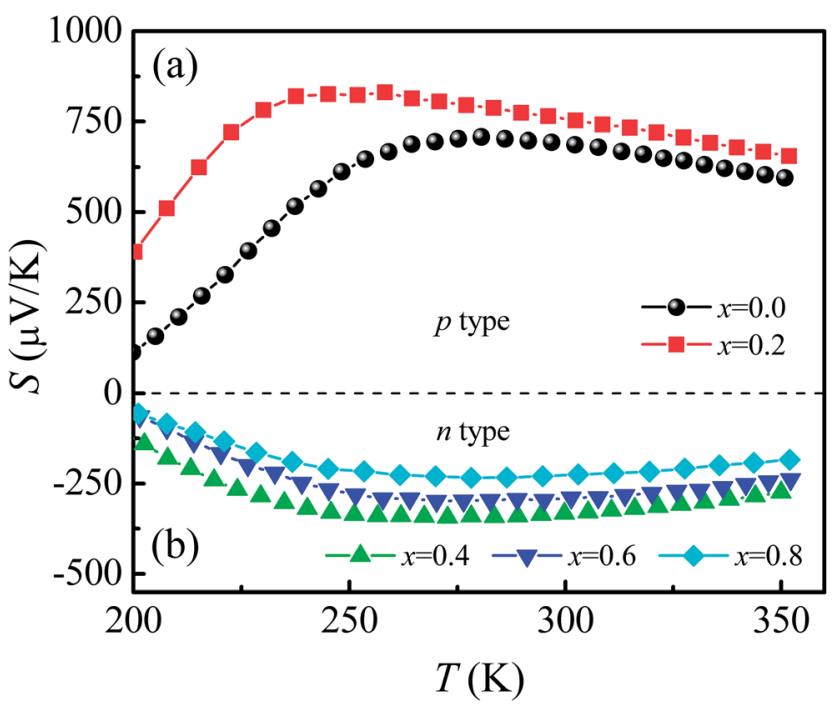

Fig. 7 (a) and (b) Temperature dependence of thermopower for $\mathrm{Ca}_{3} \mathrm{CO}_{2} \mathrm{O}_{6-x} \mathrm{~F}_{x}(x=0,0.2,0.4,0.6,0.8)$.

as $\lambda \propto \mathrm{e}^{\Theta_{\mathrm{D}} / \alpha T}$, where $\Theta_{\mathrm{D}}$ and $\alpha$ are the Debye temperature and coefficient, respectively. The fitting result shows that $\kappa$ is mainly determined by $\lambda$ in the Umklapp scattering process for this system. The values of $\Theta_{\mathrm{D}}$ obtained for the samples with $x=0.0$, $0.2,0.4,0.6$, and 0.8 are $600.1,413.8,420.6,389.8$, and $296.5 \mathrm{~K}$, respectively. In the temperature range below $T_{\mathrm{p}}$, the phononphonon scattering is weak, and $\lambda$ is determined by the lattice imperfection or the sample dimensions, and the variation of $\kappa$ is mainly determined by the specific heat, i.e., $\kappa \propto T^{3}$. So $\kappa$ decreases sharply in the low temperature range.

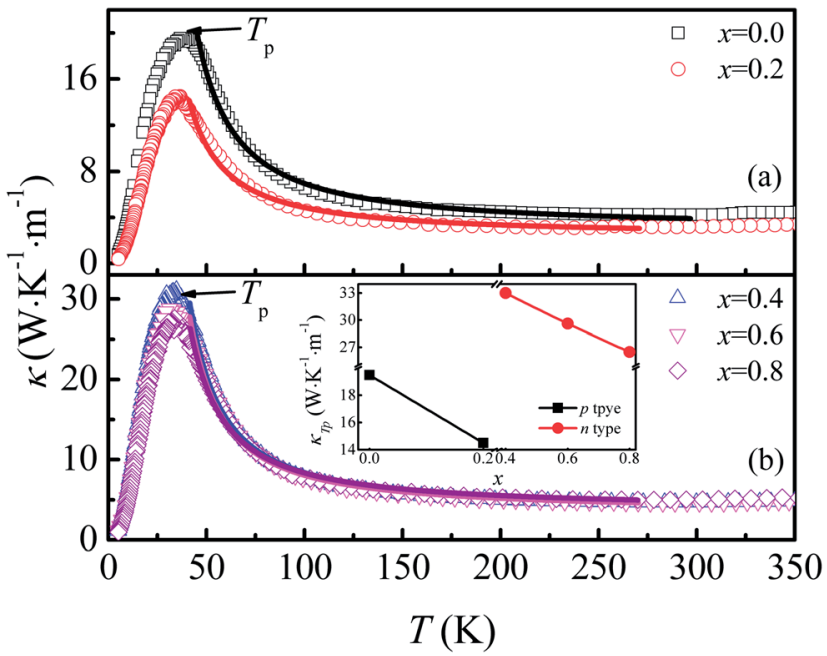

Fig. 8 (a) and (b) Temperature dependence of thermal conductivity for $\mathrm{Ca}_{3} \mathrm{CO}_{2} \mathrm{O}_{6-x} \mathrm{~F}_{x}$. Inset: thermal conductivity at $T_{p}$ as a function of $x$.

\section{Conclusions}

In summary, the structural, electrical, magnetic, and TE properties of F-doped $\mathrm{Ca}_{3} \mathrm{Co}_{2} \mathrm{O}_{6}$ were studied. The magnetic results indicate that some of the $\mathrm{Co}^{3+}$ ions at octahedral sites will change into $\mathrm{Co}^{2+}$ because of the substitution of $\mathrm{F}^{-}$for $\mathrm{O}^{2-}$, and the induced $\mathrm{Co}^{2+}$ ions are antiferromagnetically coupled with the nearest neighbor $\mathrm{Co}^{3+}$. The thermopower and Hall coefficient results show that the carrier type of the series samples can be changed from $\mathrm{p}$ - to n-type using a suitable amount of $\mathrm{F}$ doping. The room temperature thermopower changed from a positive value of $752.7 \mu \mathrm{V} \mathrm{K}^{-1}$ to a negative value of $S_{300 \mathrm{~K}}=$ $-333.1 \mu \mathrm{V} \mathrm{K}^{-1}$ as $x$ reaches 0.4 . The resistivity of both the p-type and n-type samples decreases with increasing F-doping content. The thermal conductivity of both the n-type and p-type samples decrease monotonously with increasing $x$ with a sudden increase at $x=0.4$. The sudden increase of the thermal conductivity may be related to the decreased scattering at the grain boundary and the decrease of $E_{\mathrm{a}}$ at the critical point of carrier type change.

\section{Acknowledgements}

This work is supported by the National Key Basic Research under Contract No. 2011CBA00111, and the National Nature Science Foundation of China under Contract No. U1232140, 11504380 and 11174293.

\section{Notes and references}

1 L. D. Zhao, S. H. Lo, Y. S. Zhang, H. Sun, G. J. Tan, C. Uher, C. Wolverton, V. P. Dravid and M. G. Kanatzidis, Nature, 2014, 508, 373-378.

2 I. Terasaki, Y. Sasago and K. Uchinokura, Phys. Rev. B: Condens. Matter Mater. Phys., 1997, 56, R12685-R12687. 
3 R. Funahashi and M. Shikano, Appl. Phys. Lett., 2002, 81, 1459-1461.

4 A. C. Masset, C. Michel, A. Maignan, M. Hervieu, O. Toulemonde, F. Studer, B. Raveau and J. Hejtmanek, Phys. Rev. B: Condens. Matter Mater. Phys., 2000, 62, 166-175.

5 M. Mikami, R. Funahashi, M. Yoshimura, Y. Mori and T. Sasaki, J. Appl. Phys., 2003, 94, 6579-6582.

6 H. Fjellvag, E. Gulbrandsen, S. Aasland, A. Olsen and B. C. Hauback, J. Solid State Chem., 1996, 124, 190-194.

7 E. V. Sampathkumaran, N. Fujiwara, S. Rayaprol, P. K. Madhu and Y. Uwatoko, Phys. Rev. B: Condens. Matter Mater. Phys., 2004, 70, 014437.

8 A. Maignan, V. Hardy, S. Hebert, M. Drillon, M. R. Lees, O. Petrenko, D. Mc, K. Paul and D. Khomskii, J. Mater. Chem., 2004, 14, 1231-1234.

9 K. Yamada, Z. Honda, J. Luo and H. Katori, J. Alloys Compd., 2006, 423, 188-190.

10 T. Basu, K. K. lyer, K. Singh, K. Mukherjee, P. L. Paulose and E. V. Sampathkumaran, Appl. Phys. Lett., 2014, 105, 102912.

11 S. D. Kaushik, S. Rayaprol, J. Saha, N. Mohapatra, V. Siruguri, P. D. Babu, S. Patnaik and E. V. Sampathkumaran, J. Appl. Phys., 2010, 108, 084106.

12 J. Takahashi, H. Yamane and M. Shimada, Jpn. J. Appl. Phys., 2004, 43, L331-L333.

13 J. Takahashi, M. Shimada and H. Yamane, Phys. Status Solidi A, 2006, 203, 2836-2840.

14 J. Takahashi, M. Shimada, K. Iwasaki and H. Yaname, J. Electroceram., 2006, 17, 95-98.

15 M. Senthilkumar and R. Vijayaraghavan, Sci. Technol. Adv. Mater., 2009, 10, 015007.

16 S. Hebert, D. Flahaut, C. Martin, S. Lemonnier, J. Noudem, C. Goupil, A. Maignan and J. Hejtmanek, Prog. Solid State Chem., 2007, 35, 457-467.

17 S. Rayaprol, S. D. Kaushik, K. K. lyer and E. V. Sampathkumaran, Solid State Commun., 2015, 223, 67-73.

18 S. Rayaprol and E. V. Sampathkumaran, Pramana, 2005, 65, 491-500.

19 P. L. Paulose, N. Mohapatra and E. V. Sampathkumaran, Phys. Rev. B: Condens. Matter Mater. Phys., 2008, 77, 172403.

20 H. Kageyama, K. Yoshimura, K. Kosuge, M. Azuma, M. Takano, H. Mitamura and T. Goto, J. Phys. Soc. Jpn., 1997, 66, 3996-4000.
21 R. Moubah, S. Colis, G. Schmerber, J. Petersen and A. Dinia, Appl. Phys. Lett., 2009, 94, 141907.

22 B. J. Tan, K. J. Klabunde and P. M. A. Sherwood, J. Am. Chem. Soc., 1991, 113, 855-861.

23 M. Oku and K. Hirokawa, J. Electron Spectrosc. Relat. Phenom., 1976, 8, 457-481.

24 G. Mattogno, C. Ferragina, M. A. Massucci, P. Patrono and A. La Ginestra, J. Electron Spectrosc. Relat. Phenom., 1988, 46, 285-295.

25 K. Takubo, T. Mizokawa, S. Hirata, J. Y. Son, A. Fujimori, D. Topwal, D. D. Sarma, S. Rayaprol and E. V. Sampathkumaran, Phys. Rev. B: Condens. Matter Mater. Phys., 2005, 71, 073406.

26 S. Agrestini, C. L. Fleck, L. C. Chapon, C. Mazzoli, A. Bombardi, M. R. Lees and O. A. Petrenko, Phys. Rev. Lett., 2011, 106, 197204.

27 P. Lampen, N. S. Bingham, M. H. Phan, H. Srikanth, H. T. Yi and S. W. Cheong, Phys. Rev. B: Condens. Matter Mater. Phys., 2014, 89, 144414.

28 J. Paddison, S. Agrestini, M. R. Lees, C. L. Fleck, P. P. Deen, A. L. Goodwin, J. R. Stewart and O. A. Petrenko, Phys. Rev. B: Condens. Matter Mater. Phys., 2014, 90, 014411.

29 S. Takeshita, T. Goko, J. Arai and K. Nishiyama, J. Phys. Chem. Solids, 2007, 68, 2174-2177.

30 A. Jain, S. Singh and S. M. Yusuf, Phys. Rev. B: Condens. Matter Mater. Phys., 2006, 74, 174419.

31 S. Aasland, H. Fjellvag and B. Hauback, Solid State Commun., 1997, 101, 187-192.

$32 \mathrm{H}$. Wu, M. W. Haverkort, Z. Hu, D. I. Khomskii and L. H. Tjeng, Phys. Rev. Lett., 2005, 95, 186401.

33 Y. N. Huang, B. C. Zhao, S. Lin, W. H. Song and Y. P. Sun, J. Appl. Phys., 2013, 114, 093709.

34 M. Lubini, E. Chinarro, B. Moreno, V. C. de Sousa, A. K. Alves and C. P. Bergmann, J. Phys. Chem. C, 2016, 120, 64-69.

35 Y. Wang, Y. Sui, P. Ren, L. Wang, X. J. Wang, W. H. Su and H. J. Fan, Chem. Mater., 2010, 22, 1155-1163.

36 K. Iwasaki, H. Yamane, S. Kubota, J. Takahashi and M. Shimada, J. Alloys Compd., 2003, 358, 210-215.

37 B. Fisher, L. Patlagan, G. M. Reisner and A. Knizhnik, Phys. Rev. B: Condens. Matter Mater. Phys., 2000, 61, 470-475. 